\title{
Bladder intracavitary hyperthermic perfusion chemotherapy for the prevention of recurrence of non-muscle invasive bladder cancer after transurethral resection
}

\author{
MINGCHEN BA ${ }^{1}$, SHUZHONG CUI ${ }^{1}$, BIN WANG ${ }^{1}$, HUI LONG ${ }^{2}$, ZHAOFEI YAN ${ }^{1}$, \\ SHUAI WANG $^{1}$, YINBING WU ${ }^{1}$ and YUANFENG GONG ${ }^{1}$ \\ ${ }^{1}$ Intracelom Hyperthermic Perfusion Therapy Center, Affiliated Cancer Hospital and Institute of Guangzhou \\ Medical University, Guangzhou, Guangdong 510095; ${ }^{2}$ Department of Urologic Oncology, \\ Guangzhou Dermatology Institute, Guangzhou, Guangdong 510095, P.R. China
}

Received August 31, 2016; Accepted November 21, 2016

DOI: 10.3892/or.2017.5570

\begin{abstract}
Preventing the recurrence of non-muscle invasive bladder cancer (NMIBC) post-transurethral resection (TUR) remains challenging. The aim of the present study was to investigate the effectiveness and safety of bladder intracavitary hyperthermic perfusion chemotherapy (BHPC) for prevention of NMIBC recurrence post-TUR. Between December 2006 and December 2014, 53 patients with NMIBC who underwent TUR were randomly assigned to receive BHPC (BHPC group, 28 patients) or intravesical chemotherapy alone (chemotherapy group, 25 patients) at the Intracelom Hyperthermic Perfusion Therapy Center of Guangzhou Medical University Cancer Hospital (Guangzhou, China). BHPC was performed by combining perfusion-based hyperthermia with chemotherapeutic agent mitomycin $\mathrm{C}$ (MMC) in the bladder, and the chemotherapy group of patients received bladder MMC perfusion. The concentration of MMC in the perfusion fluid and serum were assessed at different time-points. Tumor recurrence, disease-free survival (DFS), and side-effects were recorded and compared between the 2 groups. Results revealed that BHPC was performed smoothly, at $\sim 44^{\circ} \mathrm{C}$ in the bladder cavity. Patients tolerated BHPC, and no side-effects were observed. Both BHPC and intravesical chemotherapy achieved a high MMC concentration in the bladder perfusion liquid, but low MMC concentration in the serum, although serum MMC concentrations in the BHPC group were significantly higher $(\mathrm{P}<0.05)$. The tumor recurrence rate was significantly lower
\end{abstract}

Correspondence to: Dr Mingchen Ba or Dr Shuzhong Cui, Intracelom Hyperthermic Perfusion Therapy Center, Affiliated Cancer Hospital and Institute of Guangzhou Medical University, Guangzhou, Guangdong 510095, P.R. China

E-mail: bamingchen@126.com

E-mail: cuishuzhong@126.com

Key words: non-muscle invasive bladder cancer, transurethral resection, bladder hyperthermic perfusion chemotherapy, intravesical chemotherapy, mitomycin $\mathrm{C}$, recurrence
(10.7 vs. $28.0 \% ; \mathrm{P}=0.02)$ and the DFS period was significantly longer ( $37 \pm 1.2$ vs. $19 \pm 0.9$ months; $\mathrm{P}=0.001)$ in the BHPC group than in the chemotherapy group. Our results demonstrated that BHPC is safe and effective for preventing NMIBC recurrence post-TUR and prolongs DFS.

\section{Introduction}

Among patients with bladder cancer, $\sim 75 \%$ are diagnosed with non-muscle invasive bladder cancer (NMIBC) which has a much more favorable prognosis than muscle-invasive bladder cancer $(1,2)$. Transurethral resection (TUR) has been shown to be an efficient and safe method for the treatment of NMIBC. However, NMIBC recurs in $\sim 70 \%$ of patients post-TUR, and requires lifelong treatment (3-6). Although, the recurrence rate of NMIBC is significantly decreased by TUR in combination with intravesical chemotherapy, the rate of cancer progression is not diminished (7-12). Preventing recurrence of NMIBC after TUR remains challenging (13-15).

Over recent decades, there has been an increasing interest in the employment of hyperthemia at $40^{\circ} \mathrm{C}$ and above in the treatment of malignant tumors $(13,16,18-20)$. Hyperthermia may increase chemotherapeutic drug uptake, influence the intracellular distribution and metabolism of drugs, and/or inhibit repair of DNA damage in neoplastic cells, thus, improving the effect of chemotherapeutic agents $(13,16,20)$. Mitomycin C (MMC) as an alkylating cytostatic agent is commonly used to treat NMIBC. Local administration to the bladder can be effective without causing high concentrations in the serum, and thus, causing only mild side-effects $(8,12,14,16,17)$. Numerous clinical trials have shown that combining MMC administration with hypothermia can improve tumor ablation and prevent recurrence post-TUR. Numerous studies have demonstrated that the thermal enhancement of MMC killing was greater in hypoxic cells than in aerobic cells. As bladder cancer can be hypoxic, this provides a very strong rationale for the use of MMC in combination with hyperthermia (21).

Bladder intracavitary hyperthermic perfusion chemotherapy (BHPC), the combination of local hyperthermia with chemotherapy, can significantly improve the effect of 
intracavity chemotherapy, and is reported to have an additive or supra-additive effect, preventing NMIBC recurrence post-TUR (13-16,18-20). However, these previous conventional trials were based on hot water-, microwave- or radiofrequency-induced local hyperthermia, either individually or in combination with cytostatic agents, which could maintain continuous and stable treatment temperatures, but lack effective temperature control or regulation, and cannot provide uniform distribution of heat to the bladder. These inadequate devices for local heat delivery lack appropriate methods for monitoring temperature at the targets, limiting the application of hyperthermia enhanced therapy for cancer in clinical practice. Therefore, it is necessary to both continuously and stably deliver heat to bladder neoplastic sites while avoiding causing damage to surrounding normal tissues. In addition, the pharmacokinetics of chemotherapeutic agents during thermochemotherapy is also not well understood.

Over the past 6 years, we performed continuous circulatory hyperthermic intraperitoneal perfusion chemotherapy for malignant ascites using a novel 'BR-TRG-I type high-precision hyperthermic intraperitoneal perfusion treatment system' which can precisely maintain the temperature and perfusion rate of the drug solution, with satisfactory outcomes (22-25). However, conductive BHPC for bladder cancer has been rarely reported. As the bladder wall can be easily heated by irrigation with a hot isotonic fluid, bladder tumors are particularly suitable for treatment with perfusion hyperthemia $(16,18,19)$. In this prospectively randomized controlled study we performed conductive BHPC for NMIBC after TUR and evaluated its efficacy and safety.

\section{Materials and methods}

Patients. Patients with NMIBC who received TUR were prospectively recruited between December 2006 and December 2014 at the Intracelom Hyperthermic Perfusion Therapy Center of Guangzhou Medical University Cancer Hospital (Guangzhou, China). Inclusion criteria were: i) age $\geq 18$ years; ii) with single or double NMIBC sites; iii) having not received radiation therapy in the 4 weeks preceding enrollment; iv) having not received intravesical chemotherapy in the 4 weeks preceding enrollment. Exclusion criteria were: i) known or possible bladder tumors metastasized from other organs; ii) known or possible malignant bladder tumors expanding through the serosa, invading locally or metastasizing into other internal organs; iii) $\mathrm{T}_{0}$ and $\mathrm{T}_{\text {is }}$ stage bladder cancer, determined by urinary cystoscopic observation; iv) with NMIBC unsuitable for TUR; v) known or potential pregnancy; vi) active inflammation or infection; vii) other tumors. The present study was approved by the Medical Ethics Committee of the Affiliated Cancer Hospital and Institute of Guangzhou Medical University (Guangzhou, China) (no. GZMCY20080825). Written informed consent was obtained from all patients.

The clinical stages of NMIBC were assessed by cystoscopic observation of the resected tumor biopsies and determined according to the location, size and invasion depth of tumors using the TNM classification of malignant tumors (26). Bladder tumor expansion through the serosa or distal metastasis was ascertained by chest $\mathrm{x}$-ray, computerized tomography (CT) or magnetic resonance imaging (MRI) examination.
$T U R$. Under epidural anaesthesia, the bladder tumors of the patients were examined by cystoscopic observation, and then underwent TUR. The tumor profile and safe margin were initially defined, and resection was then performed carefully, avoiding bladder perforation and excessive bladder wall distention. After removal of the bulging mass, hemostasis was achieved in the base lesions and surrounding mucosa, carefully with grasp and bite as previously reported (4-6).

After TUR, 24 F 3- or 2-way Foley catheters were introduced into the bladder cavity for BHPC or intravesical chemotherapy alone, respectively and warm saline was then injected into the sac to fix the catheter in the bladder cavity. Resected specimens were reviewed by a pathologist to evaluate the depth of resection, and the histological characteristics of specimens were assessed following hematoxylin and eosin (H\&E) staining post-treatment.

BHPC procedure. BHPC was performed using our novel 'BR-TRG-I type high-precision hyperthermic intraperitoneal perfusion treatment system' (Guangzhou Bright Medical Technology Co., Ltd. Guangzhou, China), in which adaptive fuzzy control technology is principally used for precise control of the drug solution perfusion temperature $\left( \pm 0.10^{\circ} \mathrm{C}\right)$ and rate $( \pm 5 \%)(23-26)$. The device, shown in Fig. 1, has been approved by the Chinese Food Drug Administration (approval no. 2009-3260924).

BHPC was carried out 1-2 days after TUR when gross hematuria was arrested. The 'BR-TRG-I type high-precision hyperthermic intraperitoneal perfusion treatment system' was connected to pipeline systems (Guangzhou Bright Medical Technology Co., Ltd.), with a storage bag containing mitomycin C (MMC) in sterile saline. The perfusion rate was set and the therapeutic pipeline path was opened to form a circulating system. The instrument was debugged, and the treatment temperature and time were set according to the inputted clinical data of the patients (e.g., $45^{\circ} \mathrm{C}$ for $60 \mathrm{~min}$ ). The treatment temperature during BHPC was assessed by the 'BR-TRG-I type high-precision hyperthermic intraperitoneal perfusion treatment system' with the temperature probe inserted in a blind pipe within an inflated water sac, linked to an infusion tube near an infusion pipeline or in a blind pipe within an inflated water sac, linked to an outlet pipeline near a $24 \mathrm{~F} 3$-way Foley catheter (shown in Fig. 2), which was connected to an infusion pipeline and an outlet pipeline as shown in Fig. 2.

Before BHPC, the perfusion liquid was adjusted to $45^{\circ} \mathrm{C}$ within the bladder according to the perfusion pressure and the patient's subjective experience. The amount of perfusion fluid within the bladder cavity was increased or decreased accordingly, and the temperature was monitored closely using the temperature probe during the perfusion procedure. At the early stage of employing BHPC treatment using our self-developed 'BR-TRG-I type high-precision hyperthermic intraperitoneal perfusion treatment system', to assure the safety of the treatment and avoid any adverse effects of hyperthermia and high pressure in the bladder, the vital signs of the patients (including blood pressure, heart and respiratory rate, and blood oxygen saturation) were monitored using a G3HJ20025 multi-parameter patient monitor (Mindray Bio-Medical Electronics Co. Ltd., Shenzhen, China). BHPC treatment was terminated when the clinical characteristics of the patients 

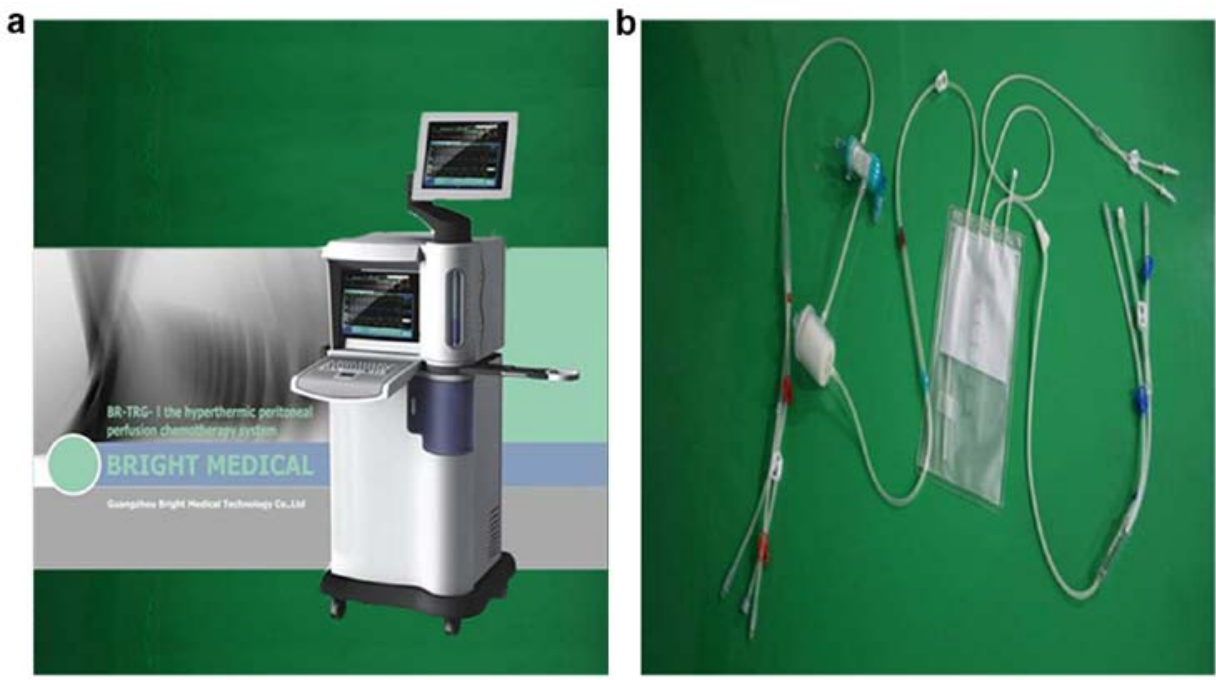

Figure 1. BR-TRG-I type high-precision hyperthermic intraperitoneal perfusion treatment system for BHPC. (a) The device system. (b) The pipelines of the system. BHPC, bladder intracavitary hyperthermic perfusion chemotherapy.

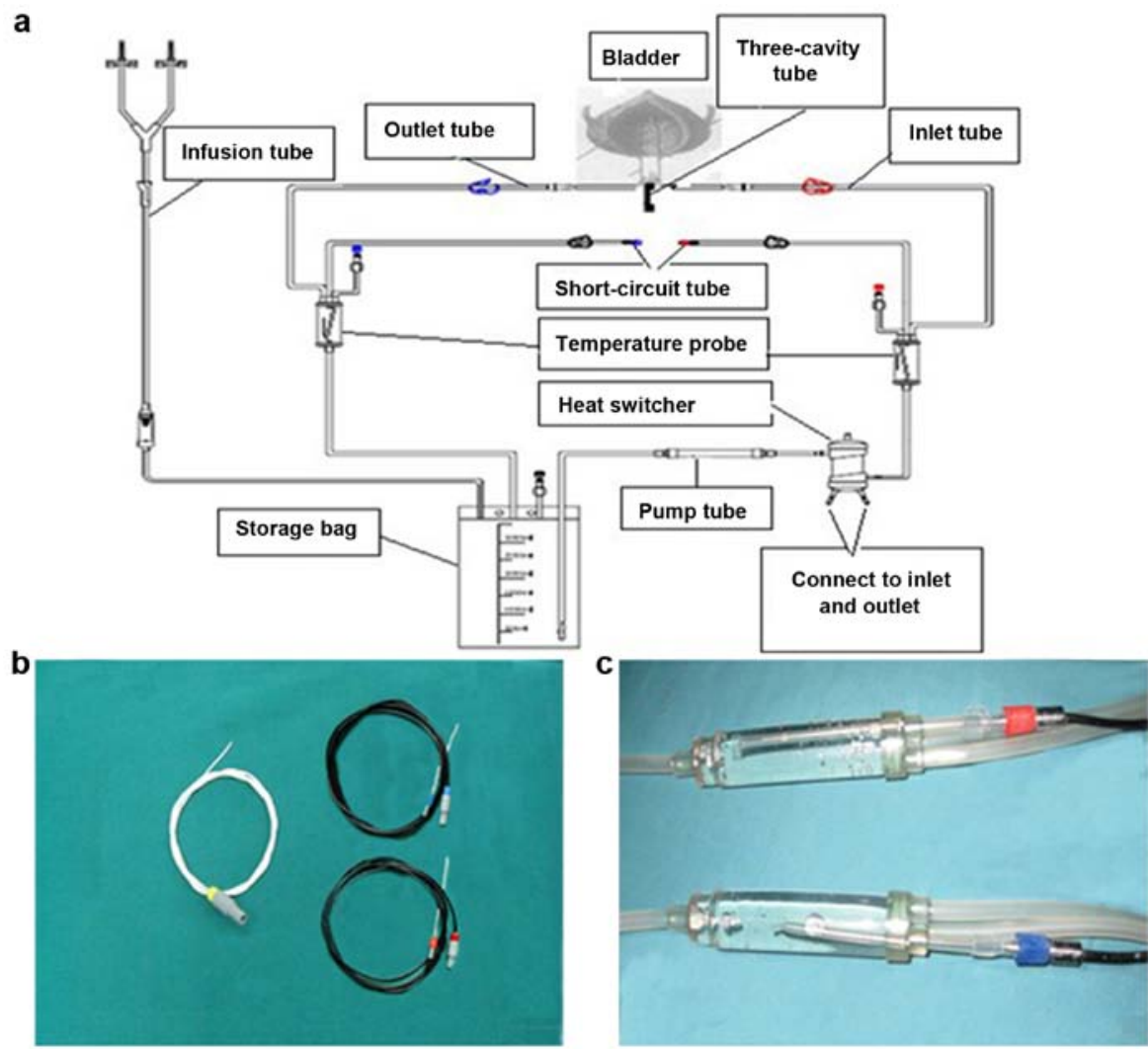

Figure 2. The schematic diagram of the pipeline system for BHPC and temperature-monitoring probes. (a) The schematic diagram of the pipeline system of BHPC. (b) Temperature-monitoring probes; one tip is put into a fixed water sac linked to an infusion tube near an inlet (red cap) or outflow catheters (blue cap) at the top of a 24 F 3-way Foley catheter (yellow cap), and another tip is connected to the device system. (c) Temperature-monitoring probe location; the red cap is located near the infusion catheters, and the blue cap is located near the outflow catheters. BHPC, bladder intracavitary hyperthermic perfusion chemotherapy.

deteriorated. BHPC was performed once a week for a total of 3 rounds for each patient. The $24 \mathrm{~F} 3$-way Foley catheter was retained for 3-5 days, thus, bleeding had to be monitored, but removed after the second or third session.

Intravesical chemotherapy. For patients in the chemotherapy group, a 24 F 2-way Foley catheter was introduced into the bladder cavity for intravesical chemotherapy after TUR. At room temperature, MMC in sterile saline was delivered to the bladder through a catheter injection syringe by way of the 24 F 2-way Foley catheter. The catheter was then closed. During the treatment, patients frequently moved, transitioning from lying in the supine or prone position, to lying on the left or right side, and sitting upright to evenly distribute the 


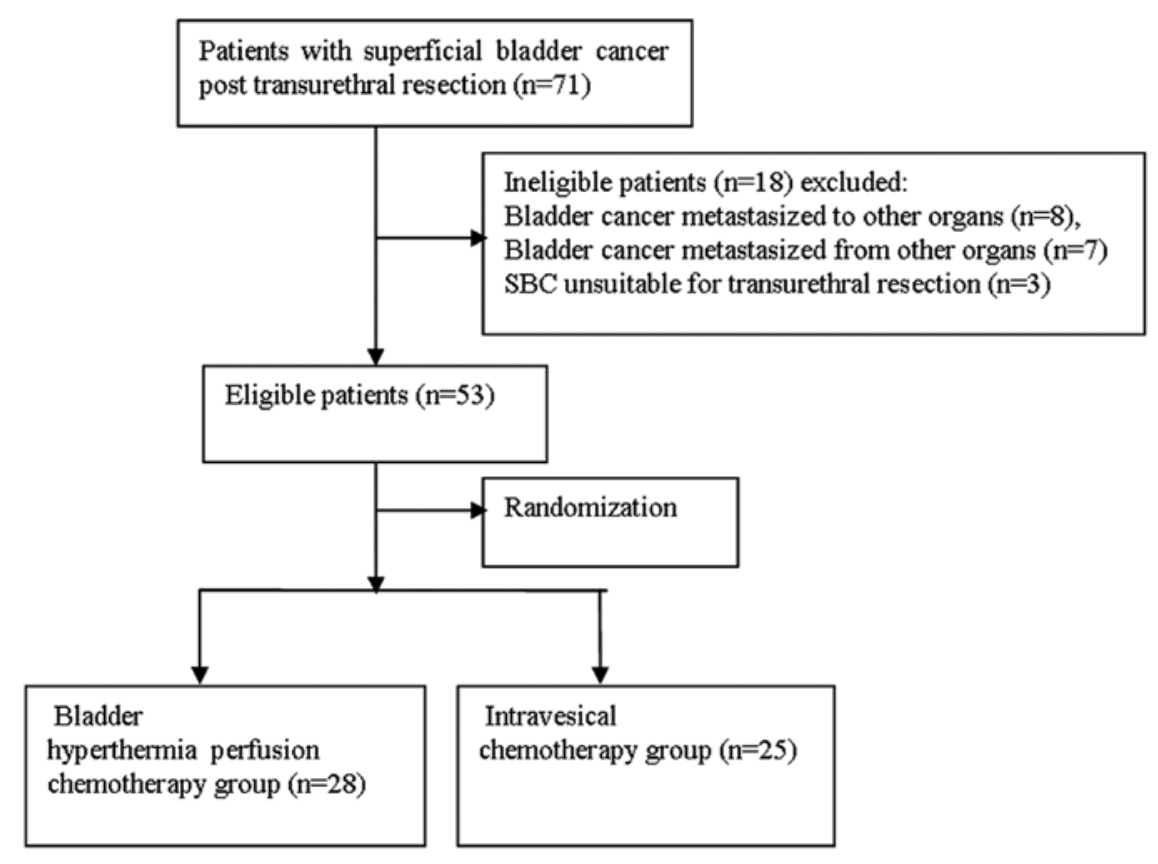

Figure 3. Flowchart of the patient recruitment for bladder intracavitary hyperthermic perfusion and intravesical chemotherapies.

therapeutic agent within the bladder cavity. After $1 \mathrm{~h}$, the MMC solution was discharged. Intravesical chemotherapy was performed once a week, for a total of 3 times for each patient, and the $24 \mathrm{~F} 2$-way Foley catheter was retained or removed as described for the BHPC group.

Pharmacokinetics of MMC. Venous blood was sampled from venous indwelling needles. Perfusion liquid samples were collected from the perfusion system short circuit outflow catheters in the BHPC group, or directly from the 24 F 2-way Foley catheter in the chemotherapy group. Perfusion liquid, serum or MMC standard samples (200 $\mu 1$, degassed prior to use) were mixed with an equal volume of acetonitrile containing $20 \mu \mathrm{g}$ diazepam as an internal standard by vortexing for $1 \mathrm{~min}$. The mixture was then centrifuged at 15,000 rpm for $15 \mathrm{~min}$ and the supernatant was collected. Aliquots of $10 \mu \mathrm{l}$ of supernatant were subjected to LC-20AB high-performance liquid chromatography (HPLC; Shimazdu, Kyoto, Japan) with Zorbax RP and $\mathrm{C}_{18}(250 \times 4.6 \mathrm{~mm}$; particle size $5 \mu \mathrm{m})$ packed columns in the stationary phase. The analysis protocol was carried out as previously described $(17,27,28)$, with some modifications. The mobile phase consisted of $50 \mathrm{mM}$ potassium dihydrogen phosphate buffer solution, acetonitrile and methanol at a ratio of 60:20:20 (\%, volume) at $\mathrm{pH} 3.0$, and was filtered through a $0.22-\mu \mathrm{m}$ membrane filter (Millipore, Billerica, MA, USA). The flow rate of the injection of samples was $1.2 \mathrm{ml} / \mathrm{min}$, and the absorption wavelength for detection was $210 \mathrm{~nm}$. The column oven temperature was maintained at $35^{\circ} \mathrm{C}$. The MMC concentration of perfusion liquid and serum were calculated according to standard curves within the linear range of $40-60 \mathrm{ng} / \mathrm{ml}$ for the perfusion liquid and $0.5-15 \mu \mathrm{g} / \mathrm{ml}$ for the serum.

Clinical follow-up and efficacy assessment. For the presence of bleeding and edema at the wound surface after the TURBT, it was difficult to see whether there were any residual tumors which were determined as the presence of the tumor remnant in the primary tumor sites under the cystoscopic observation, and thus, it could not be assured that tumors were totally resected. When the residual tumors after 3 rounds of BHPC or intravesical chemotherapy were found, a second resection was carried out, followed by the histological examination of the resected biopsies to observe the histological changes of the residual tumors after the intravesical treatment. Tumor biopsies taken before and post-treatment were stained using H\&E and graded by cystoscopic observation as previously described (4-6). Follow-up consisted of urinary cystoscopic observation at 1 month after TUR, and then quarterly thereafter for up to 1 year. Patients received abdominal and pelvic CT scans after 3, 6 and 12 months, for up to 1 year, or when were clinically indicated. One year later, follow-up was carried out every 6 months or less frequently, when the patients exhibited no signs of recurrence. Primary end points were the recurrence of tumors, disease-free survival (DFS) period and rate during the follow-up. Toxic effects of anticancer drugs were graded according to the Common Toxicity Criteria of National Cancer Institute (NCI) for Adverse Events (29).

Statistical analysis. Data were analyzed using SPSS version 19.0 (SPSS, Inc., Chicago, IL, USA). All continuous data were presented as the mean \pm standard deviation. Student's t-test was used for between-group comparisons, and paired sample t-test was used to compare results at multiple time-points between groups. DFS was analyzed using the Kaplan-Meier curve method. A P-value of $<0.05$ was considered to indicate a statistically significant result.

\section{Results}

Patient demographic and clinical characteristics. As indicated in Fig. 3, 71 eligible patients with NMIBC post-TUR were primarily recruited, and 53 were eventually enrolled in the present study. The enrolled participants included 39 male 
Table I. Characteristics of the patients with superficial bladder cancer post-transurethral resection.

\begin{tabular}{|c|c|c|c|}
\hline & BHPC group $(n=28)$ & Chemotherapy group $(n=25)$ & P-value \\
\hline Age in years, mean (range) & $50.7 \pm 1.9(37-66)$ & $51.6 \pm 2.3(39-66)$ & $>0.05$ \\
\hline \multicolumn{4}{|l|}{ Gender n $(\%)$} \\
\hline Male & $26(92.9 \%)$ & $23(92.0 \%)$ & $>0.05$ \\
\hline Female & $2(7.1 \%)$ & $2(8.0 \%)$ & $>0.05$ \\
\hline Disease courses (days) & $11.35 \pm 1.3$ & $11.27 \pm 1.6$ & $>0.05$ \\
\hline \multicolumn{4}{|l|}{ Tumor location, n (\%) } \\
\hline Side wall & $6(21.5)$ & $6(24.0)$ & $>0.05$ \\
\hline Posterior wall & $5(17.9)$ & $4(16.0)$ & $>0.05$ \\
\hline Top area & $3(10.7)$ & $2(8.0)$ & $>0.05$ \\
\hline Triangle area & $8(28.6)$ & $8(32.0)$ & $>0.05$ \\
\hline Double tumors & $6(21.5)$ & $5(20.0)$ & $>0.05$ \\
\hline \multicolumn{4}{|l|}{ Tumor size $(\mathrm{cm}), \mathrm{n}(\%)$} \\
\hline$\geq 0.5$ & $16(57.1)$ & $13(52.0)$ & $>0.05$ \\
\hline$<0.5$ & $12(50.0)$ & $12(48.0)$ & $>0.05$ \\
\hline \multicolumn{4}{|l|}{ Tumor stage, $\mathrm{n}(\%)$} \\
\hline $\mathrm{T} 1$ & $4(14.3)$ & $3(12.0)$ & $>0.05$ \\
\hline $\mathrm{T} 2$ & $24(85.7)$ & $22(88.0)$ & $>0.05$ \\
\hline
\end{tabular}

BHPC, bladder intracavitary hyperthermic perfusion chemotherapy.

and 14 female patients, with a mean age of $51.3 \pm 2.4$ years (ranging from 37 to 66 years). There were 7 patients with stage T1 and 46 with stage T2 tumors. As non-muscle invasive $\mathrm{T} 1$ and $\mathrm{T} 2$ bladder cancer can be easily resected by TURBT, which is required for BHPC treatment, T1 and T2 patients were enrolled in the present study. Usually, NMIBC can be totally resected by TURBT, with a similar efficacy to cystectomy but with less complications. BHPC following TURBT effectively prevented the postoperative recurrence of tumors. Therefore, intravesical treatment was used. According to the computer-based random distribution, patients were randomly assigned into the BHPC group (28 patients) or chemotherapy group (25 patients) (Fig. 3). In all patients gross hematuria was arrested 2 days post-TUR, but slight hematuria persisted for up to one week following the first treatment. Age, gender, disease course and tumor location, stage and size, did not significantly differ between the 2 groups (all $\mathrm{P}>0.05$; Table I).

Gross outcomes and adverse effects. BHPC and intravesical chemotherapy were successful. During BHPC the temperature of the infusion tube was stably controlled at $\sim 45^{\circ} \mathrm{C}$, an outlet pipeline at $\sim 43^{\circ} \mathrm{C}$, and the temperature in the bladder cavity at $\sim 44^{\circ} \mathrm{C}$ (Fig. 4). All patients tolerated all 3 BHPC or intravesical chemotherapy treatments, except for one case where treatment was interrupted due to severe chemical cystitis.

During BHPC or intravesical chemotherapy, patients exhibited no adverse effects except for transient fever and abdominal distension. No gastrointestinal events or bone marrow suppression were observed, and laboratory tests revealed no significant changes in blood markers, electrolyte levels or liver and kidney function after treatment in either group.
Pharmacokinetics of MMC. Although HPLC analysis indicated that the bladder perfusion MMC concentration differed significantly between the 2 groups, the MMC concentration gradually decreased during treatment in both groups, and the rate of decrease in concentration did not significantly differ between the 2 groups (Fig. 5a). The MMC concentration of the BHPC perfusion liquid group was significantly lower locally than that of the chemotherapy group ( $\mathrm{P}>0.05$; Fig. 5a). However, serum MMC concentration in the $\mathrm{BHPC}$ group during administration at a $45^{\circ} \mathrm{C}$ temperature for $1 \mathrm{~h}$ was significantly higher than that of the chemotherapy group $(\mathrm{P}<0.05$; Fig. 5b). During the first treatment session, serum MMC concentration in the BHPC group of patients did not significantly differ from the serum MMC concentration during the second or third treatment sessions in either the BHPC or chemotherapy groups, and there was no significant difference in the MMC concentration of the perfusion liquid and serum between the 2 groups ( $\mathrm{P}>0.05$; Fig. $5 \mathrm{~b}$ ).

Cystoscopic and histological observation. In the BHPC group of patients, cystoscopic observation revealed that no viable tumors remained in most patients, although, tumors remained in 3 patients. Residual tumors were observed to be covered by a grey-white slough, which extended across the bladder mucosa, and associated with congestion and edema at the mucosal surface. Inflammatory cells (sometimes including numerous eosinophils) were observed infiltrating the lamina propria, and complete necrosis was accompanied by local vascular changes (such as necrosis and thrombosis) in the small vessels of tumors, and hemorrhage into the stroma (Fig. 6a). In the chemotherapy group of patients, residual bladder tumors were observed in only 2 patients, and only congestion and edema of 


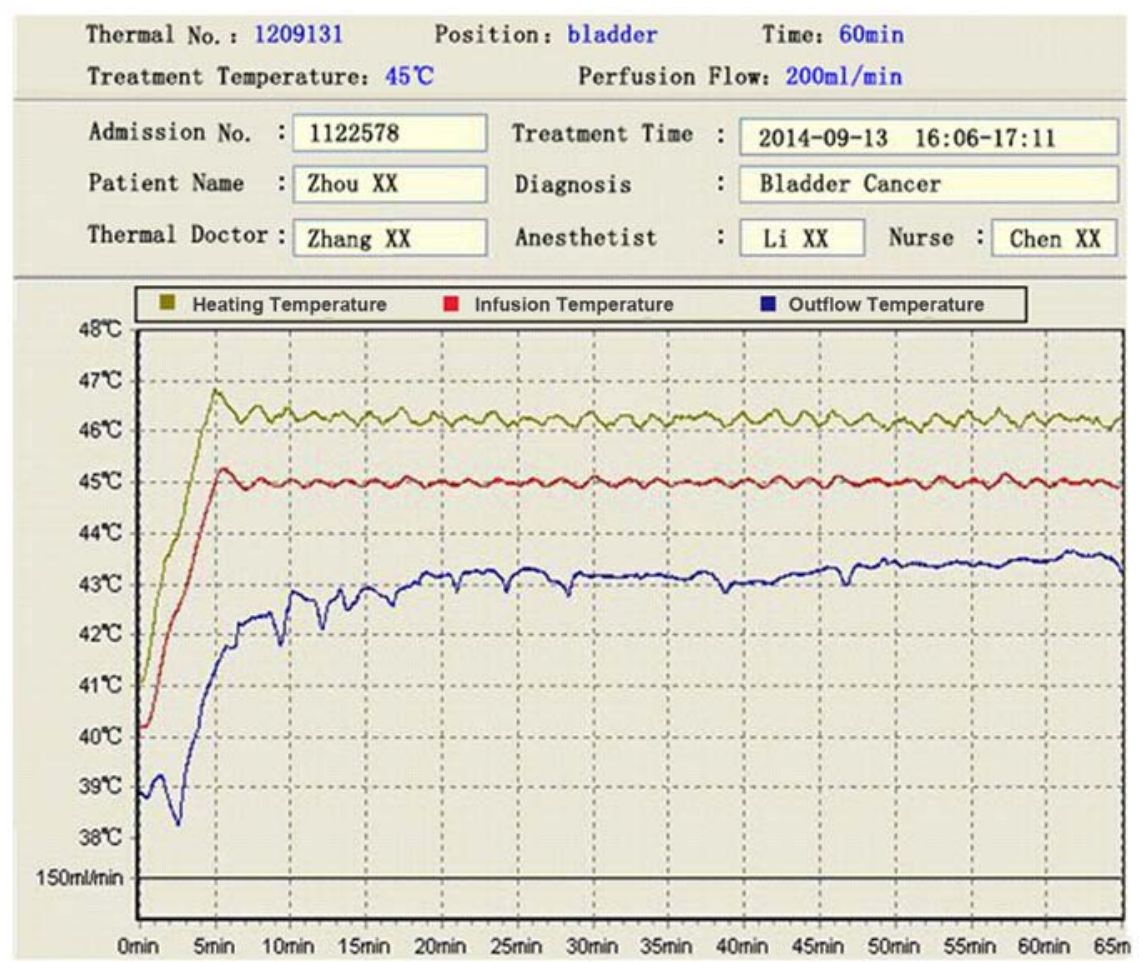

Figure 4. The temperature of the perfusion fluid in the intravesical bladder (intravesical temperature) during BHPC. BHPC, bladder intracavitary hyperthermic perfusion chemotherapy.

a

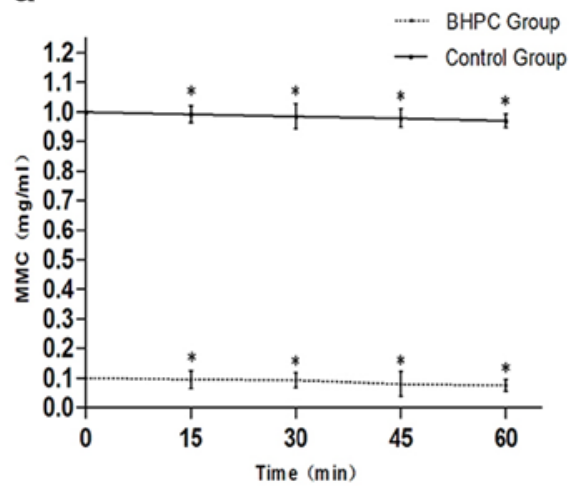

b

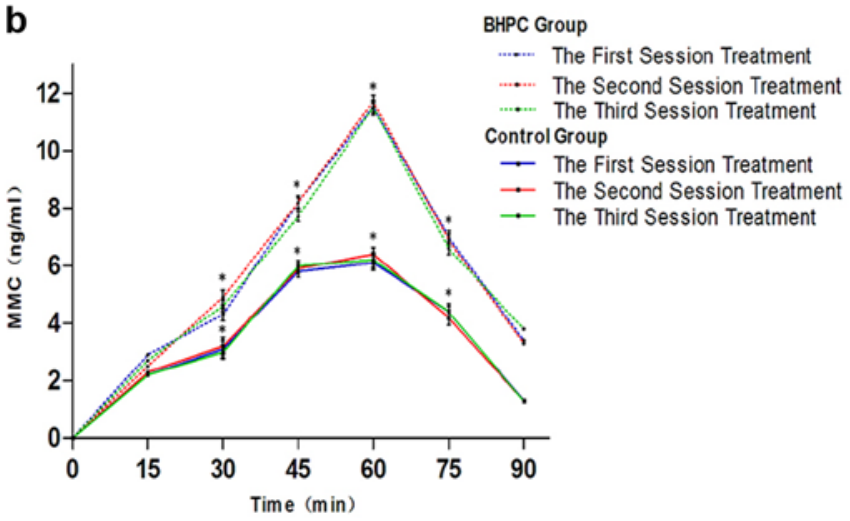

Figure 5. MMC levels in perfusion fluid and serum in the BHPC and intravesical perfusion chemotherapy groups. (a) Dynamics of MMC concentration in perfusion fluid. (b) Dynamics of MMC concentration in serum; "P<0.05. MMC, mitomycin C; BHPC, bladder intracavitary hyperthermic perfusion chemotherapy.
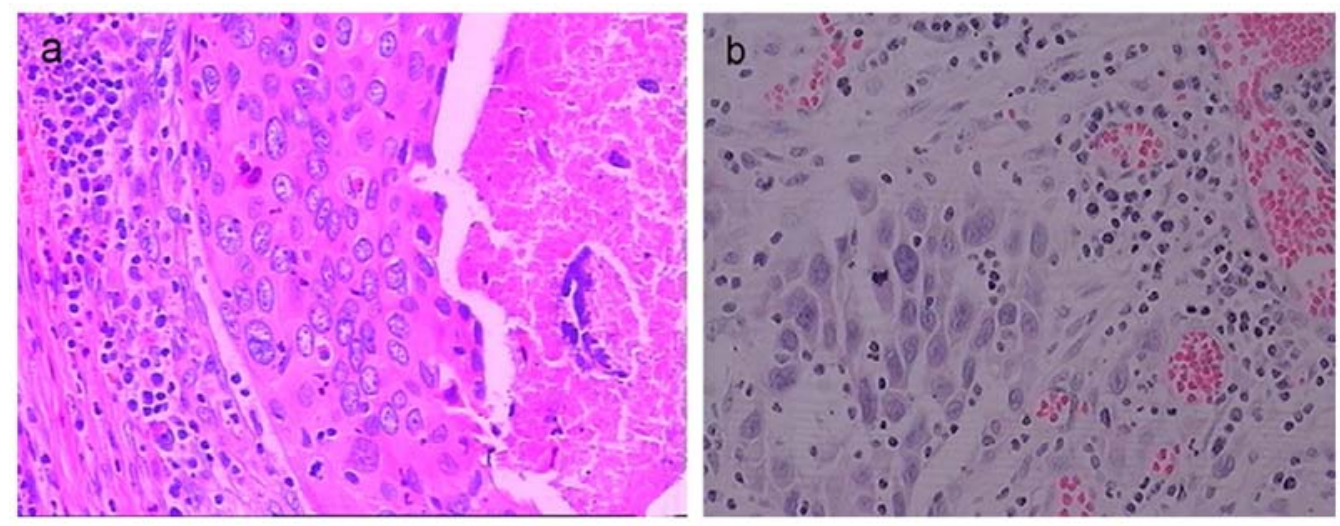

Figure 6. Histological characteristics of a residual bladder tumor after BHPC treatment and intravesical chemotherapy. (a) Histological characteristics of a bladder tumor $1 \mathrm{~h}$ after the final BHPC treatment (magnification, $\mathrm{x} 400$ ). (b) Histological characteristics of a bladder tumor $1 \mathrm{~h}$ after the final intravesical chemotherapy (magnification, $\mathrm{x} 400$ ). BHPC, bladder intracavitary hyperthermic perfusion chemotherapy. 
Table II. Clinical efficacy in patients with superficial bladder cancer post-transurethral resection treated by BHPC or intravesical chemotherapy alone.

\begin{tabular}{|c|c|c|c|c|}
\hline & $\begin{array}{c}\text { Tumor recurrence rate } \\
\% \text { (n/total) }\end{array}$ & $\begin{array}{l}\text { DFS period } \\
\text { (months) }\end{array}$ & $\begin{array}{l}\text { DFS rate } \\
(\%)\end{array}$ & $\begin{array}{c}\text { Complications } \\
\mathrm{n}\end{array}$ \\
\hline BHPC group $(n=28)$ & $10.7(3 / 28)$ & $37 \pm 1.2$ & 92.9 & 0 \\
\hline Chemotherapy group $(n=25)$ & $28.0(7 / 25)$ & $19 \pm 0.9$ & 72.0 & 1 \\
\hline P-value & 0.02 & 0.001 & $<0.001$ & \\
\hline
\end{tabular}

BHPC, bladder intracavitary hyperthermic perfusion chemotherapy; DFS, disease-free survival.

the bladder mucosa except for one case complicated with severe chemical cystitis was noted. In both patients with residual bladder tumors, the bladder mucosa appeared healthy around the residual tumors. Inflammatory cells (sometimes including numerous eosinophils) were observed to infiltrate the lamina propria, but in the absence of local vascular changes or hemorrhage they were observed to infiltrate the stroma (Fig. 6b).

Follow-up and prognosis. Patients were followed-up for $47 \pm 2.3$ months (range 8-78 months). The follow-up period did not significantly differ between the 2 groups (BHPC group $47 \pm 2.7$ months vs. chemotherapy group $46 \pm 1.9$ months; $\mathrm{P}>0.05)$. All patients were reported to be living well. In patients of the BHPC group, cystoscopic observation revealed recurrence of tumors in the bladder cavity of 3 patients at 14,17 and 19 months post-BHPC. The tumor recurrence rate was $10.7 \%$ (3 out of 28 patients), the mean DFS period was $37 \pm 1.2$ months (ranging from 8 to 78 months), and the DFS rate was $92.9 \%$ during the follow-up (Table II). In contrast, in the chemotherapy group, cystoscopic observation revealed recurrence of the tumors in the bladder cavity of 7 patients at 6,13 , 13, 15, 16, 21 and 27 months after chemotherapy. The tumor recurrence rate was $28.0 \%$ ( 7 out of 25 patients), the mean DFS period was $19 \pm 0.9$ months (ranging from 6 to 54 months), and the DFS rate was $72.0 \%$ during follow-up (Table II). The tumor recurrence rate was significantly lower while the DFS period and DFS rate were significantly higher in the BHPC group than in the chemotherapy group (all $\mathrm{P}<0.05$; Table II).

\section{Discussion}

Due to the unique location and anatomic structure of the urinary bladder, bladder tumors are highly suitable for local hyperthermia combined with intravesical chemotherapy $(11,12,14-16,18,19)$. Thermochemotherapy combining the direct antitumorigenic effect of local hyperthermia with a potentiated antitumorigenic cytostatic agent improves chemotherapy outcomes $(19,30)$. However, conventional thermochemotherapy procedures do not allow precise and stable temperature control at the lesion sites, which hampers treatment efficacy (31-34). We developed a 'BR-TRG-I type high-precision hyperthermic intraperitoneal perfusion treatment system', which allows precise automatic control of the temperature and perfusion rate of the drug solution. This system includes an automatic cooling function and adaptive fuzzy control technology for precise control of the perfusion temperature and rate, and thus, control of the local temperature in the bladder cavity of NMIBC patients (22-25). To effectively kill the residual cancer cells within the bladder as well as to kill the cancer cells dropped from the bladder cavity and adhered to the bladder wall, BHPC using this device was performed immediately after the TUR of bladder tumors instead of weeks later. In the present study, we demonstrated that BHPC was successfully carried out $(33,34)$, and the rate of side-effects was similar to previously published studies (31-34). This could effectively help to stop bleeding at the wound surface and prevent the tumor recurrence after TURBT.

In the present study, we performed a randomized controlled trial to evaluate the treatment efficacy of BHPC. Since BHPC involves perfusion chemotherapy in addition to local hyperthermia, we used intravesical chemotherapy instead of simple conventional systematic chemotherapy as the control. BHPC significantly decreased tumor recurrence and increased the DFS period and rate for NMIBC patients post-TUR when compared with intravesical chemotherapy alone. This result is consistent with a previous study that indicates the advantage of conventional intravesical thermochemotherapy over intravesical chemotherapy alone (31). We attribute this effect to the synergistic effect of bladder hyperthermia and chemotherapy. In this technique, the bladder mucosa is fully extended by a large volume of BHPC, which allows chemotherapeutics better access to the remnant bladder tumor.

MMC can be systemically absorbed via intravesical administration. Numerous studies in humans have indicated that plasma concentrations after normal doses do not reach a critical toxic value (21). In the present study, both BHPC and intravesical chemotherapy achieved high local drug concentrations in the perfusion liquid, thus, at the intravesical cavity of the bladder, but serum concentrations of MMC were maintained below those associated with systematic administration of the drug. Although, the serum MMC concentrations in the BHPC group were significantly higher than those in the intravesical perfusion chemotherapy group in the present study, it did not reach sufficiently high levels to cause serious adverse effects $(21,34,35)$. The advantages of BHPC over single intravesical chemotherapy can be attributed to the synergy of local hyperthermia treatment and chemotherapy.

In the present study, the perfusion fluid MMC concentration gradually decreased during BHPC or chemotherapy treatment, perhaps due to absorption or the dilution by urine 
during treatment. As indicated by the higher serum concentration, patients in the BHPC group absorbed more MCC, indicating that thermal treatment may increase the absorption of chemotherapeutic drugs, however, MMC was diluted more significantly with urine in the intravesical chemotherapy group, and overall the decrease in perfusion liquid MCC concentration did not differ significantly between the 2 groups.

HPLC analysis indicated that the BHPC group perfusion liquid contained a significantly lower MMC concentration than that of the chemotherapy group. However, serum MMC concentration in the BHPC group was significantly higher than in the chemotherapy group when administered at $45^{\circ} \mathrm{C}$ for $1 \mathrm{~h}$. This may be attributable to the fact that local hyperthermia induced by BHPC increases the chemotherapeutic drug intake of tumor cells, drug intracellular distribution and metabolism, which may in turn increase drug release into the serum from tumor cells. This may also be associated with the full extension of the bladder wall (induced by the large volume of thermal perfusion liquid) increasing the absorptive area of bladder mucosa. In the present study, the MMC concentration of the perfusion liquid and serum did not differ significantly between the 2 groups $(\mathrm{P}>0.05)$, or between the first and second, or third BHPC treatment. This result suggested that bladder mucosal wound by TUR of bladder carcinoma did not increase the absorption of chemotherapeutic drugs. In addition, the plasma half-life of MMC is $30-50 \mathrm{~min}(34,35)$, and we found that the serum MMC concentration remained stable during BHPC, but rapidly declined after BHPC. We hypothesized that absorption of chemotherapeutic drugs compensated for metabolism of chemotherapeutic drugs during BHPC

The aim of BHPC treatment post-TUR is to cause maximal killing of residual tumors, with minimal damage to normal tissues. In the present study, the whole bladder mucosa was covered by a grey-white slough post-BHPC, with congestion and edema at the mucosa surface and with inflammatory cell (e.g. eosinophils) infiltration in the lamina propria. However these features resolved 2 months post-BHPC. This result revealed that $\mathrm{BHPC}$ is safe, targets tumor cells precisely, and causes minimal damage to the normal bladder mucosa. Tumor cell necrosis causes a shedding on the surface bladder wall, and has no significant adverse impact on the normal bladder mucosa. In previous studies, histological changes were observed after the local hyperthermia treatment, including nuclear vacuolation and pyknosis, cytoplasmic vacuolation and decrease of epithelial adhesion $(19,20,30)$. These histological changes reflected damage to the bladder cancer caused by hyperthermia treatment. In the present study, cystoscopic and histological observations revealed grey-white slough covering the whole bladder mucosa around residual tumors in the BHPC group of patients, while in contrast in the intravesical chemotherapy group of patients, the NMIBC mucosa appeared healthy in all but one patient complicated with severe chemical cystitis.

Previous studies showed that dermal vessels were far more responsive to temperature changes than normal epithelial cells, and that severe and persistent vascular reactions were often elicited by prolonged episodes of low intensity hyperthermia that fail to harm the epidermis $(13,16,20)$. Thermal effects upon blood vessels of the mucosa led to exudation of plasma and sometimes interstitial hemorrhage. These vascular changes may exacerbate direct thermal injury of the lamina propria, sometimes resulting in areas of necrosis with exfoliation of epithelium and subsequent formation of hypervascular inflamed granulation tissue $(13,16,20)$. Our results indicate complete necrosis accompanied by local vascular changes (such as necrosis and thrombosis) in the small vessels of tumors, and hemorrhage into the stroma in the BHPC group. While in the intravesical chemotherapy group, no local vascular changes were observed in the small vessels of tumors, and also no hemorrhage into the stroma were observed. These vascular changes may be partially responsible for preventing NMIBC recurrence since tumor vessels are more susceptible to thermal injury than normal tissue vessels after hyperthermia. These histological changes suggest that BHPC may be more effective in killing tumor cells in residual tumors, inhibiting tumor angiogenesis and blocking mucosal blood supply of residual tumors in bladder cancer recurrence post-TUR than intravesical chemotherapy. This result is consistent with some similar studies $(13,20)$.

BHPC using the device 'BR-TRG-I type high-precision hyperthermic intraperitoneal perfusion treatment system' in the present study was first developed by us. According to our previous experience 3 rounds of HIPEC was used to treat abdominal tumors in order to get good outcomes (22-25), consequently, BHPC was also conducted for 3 rounds in the present study. Nevertheless, for our self-developed device-based BHPC, the chemotherapeutic types of drugs and dosages involved, the treatment time and temperature are still being explored.

As 'BR-TRG-I type high-precision hyperthermic intraperitoneal perfusion treatment system' was first used in BHPC during the study period, it was not easy to recruit numerous patients who were willing to receive BHPC using this device in the randomized controlled study. Therefore, a limitation of the present study was that the sample size was relatively small. In the future we will try to enroll more patients to validate these results. Meanwhile, since the bladder wall became thinner during the perfusion of liquid, it was not practical to detect the temperature in the bladder wall or within the bladder tissue, consequently we were only able to detect the internal temperature of the infusion pipeline and outflow pipeline from the bladder cavity, which cannot guarantee an accurate reading of the temperature of the bladder mucosa due to the fact that heat conduction and undermucosal blood flow may promote loss of heat. Thus, the temperature of the bladder wall in contact with the mucosa and the mucosa itself was likely lower than the temperature in the infusion pipeline and bladder cavity. From Fig. 4 displaying the temperature of the perfusion fluid during BHPC, it can be seen that the intravesical temperature ranged from 43 to $45^{\circ} \mathrm{C}$. In a pilot animal study (data not shown), we demonstrated that during $\mathrm{BHPC}$ in pig and rabbits, the temperature $\left(42-43^{\circ} \mathrm{C}\right)$ at the serosal surface, outmost layer of the bladder wall, was a bit lower $\left(2-3^{\circ} \mathrm{C}\right)$ than that $\left(44-44.5^{\circ} \mathrm{C}\right)$ in the bladder cavity. Therefore, the temperature reaching the bladder wall and tissues was still high enough to reach the treatment temperature level (over $40^{\circ} \mathrm{C}$ ). Based on the animal study, we set the perfusion temperature at $45^{\circ} \mathrm{C}$, and similarly, in the bladder wall and tissues a temperature still higher than $40^{\circ} \mathrm{C}$ would be reached thus effectively killing the residual cancer cells 
establishing an effective treatment temperature for tumor hyperthermic therapy.

During hyperthermic intraperitoneal chemotherapy, widely employed in the treatment of ovarian cancer and malignant ascites with satisfactory outcomes (22-25), the temperature probes sensed the temperature of the perfusion liquid or within the abdominal cavity instead of the tumor tissues directly. However, this did not hamper hyperthermia in the cancer tissues. Similarly, during BHPC for patients in the present study, although the temperatures in the bladder wall and tissues were not directly assessed, the perfusion temperature of $45^{\circ} \mathrm{C}$ reaching the bladder wall and cancer tissues was estimated to be over $40^{\circ} \mathrm{C}$, i.e. the treatment temperature level, although there was some heat loss during conduction and heat removal by blood vessels. Therefore, this treatment was defined as bladder intracavitary hyperthermia and the bladder wall received a hyperthermic temperature.

In conclusion, BHPC is a feasible, safe and promising approach for the treatment of patients with NMIBC who undergo TUR, capable of decreasing the NMIBC recurrence rate, and prolonging the NMIBC recurrence interval time and increasing the DFS rate and period.

\section{Acknowledgements}

The present study was supported by the Breakthroughs in Key Areas of Guangdong and Hong Kong Projects (no. 2006Z1-E6041), the Guangdong Provincial Science and Technological Programs (no. 2009A030301013), and the Guangdong Province Natural Science Foundation Programs (no. 2014A030313494).

\section{References}

1. Siegel R, Naishadham D and Jemal A: Cancer statistics, 2012. CA Cancer J Clin 62: 10-29, 2012.

2. Hızlı F, Argun G, Güney I, Güven O, Arık AI, Başay S, Günaydın H, Başar H and Köşüş A: Obturator nerve block transurethral surgery for bladder cancer: Comparison of inguinal and intravesical approaches: Prospective randomized trial. Ir J Med Sci 185: 555-560, 2016.

3. Aziz A, Gierth M, Rink M, Schmid M, Chun FK, Dahlem R, Roghmann F, Palisaar RJ, Noldus J, Ellinger J, et al; PROMETRICS 2011 Research Group: Optimizing outcome reporting after radical cystectomy for organ-confined urothelial carcinoma of the bladder using oncological trifecta and pentafecta. World J Urol 33: 1945-1950, 2015.

4. Hermann GG, Mogensen K, Lindvold LR, Haak CS and Haedersdal M: Office-based transurethral devascularisation of low grade non-invasive urothelial cancer using diode laser. A feasibility study. Lasers Surg Med 47: 620-625, 2015.

5. Stein JP, Lieskovsky G, Cote R, Groshen S, Feng AC, Boyd S, Skinner E, Bochner B, Thangathurai D, Mikhail M, et al: Radical cystectomy in the treatment of invasive bladder cancer: Long-term results in 1,054 patients. J Clin Oncol 19: 666-675, 2001 .

6. Takai T, Inamoto T, Komura K, Yoshikawa Y, Uchimoto T, Saito K, Tanda N, Kouno J, Minami K, Uehara H, et al: Feasibility of photodynamic diagnosis for challenging TUR-Bt cases including muscle invasive bladder cancer, BCG failure or 2nd-TUR. Asian Pac J Cancer Prev 16: 2297-2301, 2015.

7. Lamm DL, Riggs DR, Traynelis CL and Nseyo UO: Apparent failure of current intravesical chemotherapy prophylaxis to influence the long-term course of superficial transitional cell carcinoma of the bladder. J Urol 153: 1444-1450, 1995.

8. Newling D: Intravesical therapy in the management of superficial transitional cell carcinoma of the bladder: The experience of the EORTC GU Group. Br J Cancer 61: 497-499, 1990.
9. Rawls WH, Lamm DL, Lowe BA, Crawford ED, Sarosdy MF, Montie JE, Grossman HB and Scardino PT: Fatal sepsis following intravesical bacillus Calmette-Guerin administration for bladder cancer. J Urol 144: 1328-1330, 1990.

10. Hayne D, Stockler M, McCombie SP, Chalasani V, Long A, Martin A, Sengupta S and Davis ID: BCG+MMC trial: Adding mitomycin $\mathrm{C}$ to $\mathrm{BCG}$ as adjuvant intravesical therapy for high-risk, non-muscle-invasive bladder cancer: A randomised phase III trial (ANZUP 1301). BMC Cancer 15: 432, 2015.

11. Kamat AM, Briggman J, Urbauer DL, Svatek R, Nogueras González GM, Anderson R, Grossman HB, Prat F and Dinney CP: Cytokine panel for response to intravesical therapy (CyPRIT): Nomogram of changes in urinary cytokine levels predicts patient pesponse to bacillus Calmette-Guérin. Eur Urol 69: 197-200, 2016.

12. Ferlazzo G, Magno C, Lupo G, Rizzo M, Iemmo R, Semino C and Melioli G: A phase I study of intravesical continuous perfusion of recombinant interleukin-2 in patients with superficial bladder cancer. Am J Clin Oncol 18: 100-104, 1995.

13. Colombo R, Da Pozzo LF, Lev A, Freschi M, Gallus G and Rigatti P: Neoadjuvant combined microwave induced local hyperthermia and topical chemotherapy versus chemotherapy alone for superficial bladder cancer. J Urol 155: 1227-1232, 1996.

14. Geijsen ED, de Reijke TM, Koning CC, Zum Vörde Sive Vörding PJ, de la Rosette JJ, Rasch CR, van Os RM and Crezee J: Combining mitomycin C and regional $70 \mathrm{MHz}$ hyperthermia in patients with nonmuscle invasive bladder cancer: A pilot study. J Urol 194: 1202-1208, 2015.

15. Colombo R, Salonia A, Da Pozzo LF, Naspro R, Freschi M, Paroni R, Pavone-Macaluso M and Rigatti P: Combination of intravesical chemotherapy and hyperthermia for the treatment of superficial bladder cancer: Preliminary clinical experience. Crit Rev Oncol Hematol 47: 127-139, 2003.

16. Gofrit ON, Shapiro A, Pode D, Sidi A, Nativ O, Leib Z, Witjes JA, van der Heijden AG, Naspro R and Colombo R: Combined local bladder hyperthermia and intravesical chemotherapy for the treatment of high-grade superficial bladder cancer. Urology 63: 466-471, 2004.

17. Milla P, Fiorito C, Soria F, Arpicco S, Cattel L and Gontero P: Intravesical thermo-chemotherapy based on conductive heat: A first pharmacokinetic study with mitomycin $C$ in superficial transitional cell carcinoma patients. Cancer Chemother Pharmacol 73: 503-509, 2014.

18. Uchibayashi T, Nakajima K, Hisazumi H, Nishino A and Miyoshi N: Hyperthermic intravesical peplomycin perfusion treatment for bladder cancer. Br J Urol 72: 65-67, 1993.

19. Owusu RA, Abern MR and Inman BA: Hyperthermia as adjunct to intravesical chemotherapy for bladder cancer. Biomed Res Int 2013: 262313, 2013.

20. Ekin RG, Akarken I, Cakmak O, Tarhan H, Celik O, Ilbey YO, Divrik RT and Zorlu F: Results of intravesical chemo-hyperthermia in high-risk non-muscle invasive bladder cancer. Asian Pac J Cancer Prev 16: 3241-3245, 2015.

21. Paroni R, Salonia A, Lev A, Da Pozzo LF, Cighetti G, Montorsi F, Rigatti P and Colombo R: Effect of local hyperthermia of the bladder on mitomycin $\mathrm{C}$ pharmacokinetics during intravesical chemotherapy for the treatment of superficial transitional cell carcinoma. Br J Clin Pharmacol 52: 273-278, 2001.

22. Ba M, Long H, Zhang X, Tang Y, Wu Y, Yu F, Wang S and Cui S: Different sequential approaches of cytoreductive surgery and hyperthermic intraperitoneal chemotherapy in treating ovarian cancer with malignant ascites. J Cancer Res Clin Oncol 140: 1497-1506, 2014.

23. Ba MC, Long H, Cui SZ, Tang YQ, Wu YB, Zhang XL, Tang HS and Bai SX: Multivariate comparison of B-ultrasound guided and laparoscopic continuous circulatory hyperthermic intraperitoneal perfusion chemotherapy for malignant ascites. Surg Endosc 27: 2735-2743, 2013.

24. Ba MC, Cui SZ, Lin SQ, Tang YQ, Wu YB, Wang B and Zhang XL: Chemotherapy with laparoscope-assisted continuous circulatory hyperthermic intraperitoneal perfusion for malignant ascites. World J Gastroenterol 16: 1901-1907, 2010.

25. Cui S, Ba M, Tang Y, Liu J, Wu Y, Wang B, Zhang X, Tang H and Zhong S: B ultrasound-guided hyperthermic intraperitoneal perfusion chemotherapy for the treatment of malignant ascites. Oncol Rep 28: 1325-1331, 2012.

26. Wittekind C: 2010 TNM system: On the 7th edition of TNM classification of malignant tumors. Pathologe 31: 331-332, 2010 (In German). 
27. Dwivedi R, Singh M, Kaleekal T, Gupta YK and Tripathi M: Concentration of antiepileptic drugs in persons with epilepsy: A comparative study in serum and saliva. Int J Neurosci 126 972-978, 2016.

28. Abdelaleem EA, Naguib IA, Zaazaa HE and Hussein EA: Development and validation of HPLC and HPTLC methods for determination of cefoperazone and its related impurities. J Chromatogr Sci 54: 179-186, 2016.

29. Chen AP, Setser A, Anadkat MJ, Cotliar J, Olsen EA, Garden BC and Lacouture ME: Grading dermatologic adverse events of cancer treatments: The Common Terminology Criteria for Adverse Events Version 4.0. J Am Acad Dermatol 67: 1025-1039, 2012.

30. Lammers RJ, Witjes JA, Inman BA, Leibovitch I, Laufer M, Nativ $\mathrm{O}$ and Colombo R: The role of a combined regimen with intravesical chemotherapy and hyperthermia in the management of non-muscle-invasive bladder cancer: A systematic review. Eur Urol 60: 81-93, 2011.

31. Inman BA, Stauffer PR, Craciunescu OA, Maccarini PF, Dewhirst MW and Vujaskovic Z: A pilot clinical trial of intravesical mitomycin- $C$ and external deep pelvic hyperthermia for non-muscle-invasive bladder cancer. Int J Hyperthermia 30: 171-175, 2014.
32. Soria F, Milla P, Fiorito C, Pisano F, Sogni F, Di Marco M, Pagliarulo V, Dosio F and Gontero P: Efficacy and safety of a new device for intravesical thermochemotherapy in non-grade 3 BCG recurrent NMIBC: A phase I-II study. World J Urol 34: 189-195, 2016.

33. Sousa A, Piñeiro I, Rodríguez S, Aparici V, Monserrat V, Neira $P$, Carro E, Murias C and Uribarri C: Recirculant hyperthermic IntraVEsical chemotherapy (HIVEC) in intermediate-high-risk non-muscle-invasive bladder cancer. Int J Hyperthermia 32: 374-380, 2016.

34. Colombo R, Salonia A, Leib Z, Pavone-Macaluso M and Engelstein D: Long-term outcomes of a randomized controlled trial comparing thermochemotherapy with mitomycin- $\mathrm{C}$ alone as adjuvant treatment for non-muscle-invasive bladder cancer (NMIBC). BJU Int 107: 912-918, 2011.

35. van der Heijden AG and Dewhirst MW: Effects of hyperthermia in neutralising mechanisms of drug resistance in non-muscleinvasive bladder cancer. Int J Hyperthermia 32: 434-445, 2016. 